La Revue

des Droits

de l'Homme

\section{La Revue des droits de l'homme}

Revue du Centre de recherches et d'études sur les droits fondamentaux

$21 \mid 2022$

Revue des droits de l'homme - N²1

\title{
Les stratégies de gestion des conflits constitutionnels par les cours constitutionnelles d'Europe centrale et orientale
}

\section{Antonin Gelblat}

\section{(2) OpenEdition}

\section{Journals}

Édition électronique

URL : https://journals.openedition.org/revdh/14035

DOI : $10.4000 /$ revdh. 14035

ISSN : 2264-119X

Éditeur

Centre de recherches et d'études sur les droits fondamentaux

Référence électronique

Antonin Gelblat, « Les stratégies de gestion des conflits constitutionnels par les cours constitutionnelles d'Europe centrale et orientale », La Revue des droits de l'homme [En ligne], 21 | 2022 mis en ligne le 17 janvier 2022, consulté le 27 septembre 2022. URL : http://journals.openedition.org/ revdh/14035; DOI : https://doi.org/10.4000/revdh.14035

Ce document a été généré automatiquement le 27 septembre 2022.

Tous droits réservés 


\title{
Les stratégies de gestion des conflits constitutionnels par les cours constitutionnelles d'Europe centrale et orientale
}

\author{
Antonin Gelblat
}

1 La notion de conflit implique une situation relationnelle, la rencontre intentionnelle, le heurt ou le choc de forces antagonistes; l'opposition d'éléments, d'intérêts ou de sentiments contraires. Il est d'ailleurs synonyme de guerre, ou de lutte armée lorsqu'il vise à décrire une relation entre États. La notion semble donc entretenir des liens étroits avec celle de stratégie qui renvoie, étymologiquement, au fait de conduire une armée et qui désigne, au sens large, une méthode d'organisation de l'action en vue d'atteindre un résultat prédéterminé ou plus précisément «un ensemble cohérent de décisions que se propose de prendre un agent assumant des responsabilités, face aux diverses éventualités qu'il est conduit à envisager, tant du fait des circonstances extérieures qu'en vertu d'hypothèses portant sur le comportement d'autres agents intéressés par de telles décisions $»^{1}$. La résolution des conflits est d'ailleurs pensée comme le résultat des stratégies déployées par les agents impliqués dans une telle relation pour y faire face. Pourtant, si la notion de conflit a été transposée à la matière constitutionnelle, si le conflit constitutionnel s'est imposé, la figure du stratège est quant à elle rarement utilisée pour désigner leurs protagonistes, et singulièrement le juge constitutionnel. Ce dernier est davantage envisagé comme un arbitre ou comme une victime des conflits constitutionnels ${ }^{2}$.

2 La figure de l'arbitre a souvent été mobilisée pour définir la position du juge constitutionnel à l'égard des conflits d'interprétation de la Constitution. Cette idée a été tout particulièrement défendue s'agissant des démocraties dites « fragiles » ou " émergentes $»^{3}$. La mise en place de puissantes cours constitutionnelles dans les États d'Europe centrale et orientale à la chute du communisme répondait à cette logique ${ }^{4}$. C'est en ce sens que la Cour constitutionnelle de Hongrie a pu être présentée dans les 
années quatre-vingt-dix comme "l'une des juridictions les plus puissantes au monde » au point d'assimiler le régime hongrois à une "courtocratie " ${ }^{5}$. L'octroi de telles compétences était justifié par la nécessité de prévenir des conflits constitutionnels qui seraient plus susceptibles de se produire dans ces États conduisant un processus de transition démocratique que dans les démocraties plus établies. Ainsi, la mise en place de cours constitutionnelles dotées des capacités d'intervenir dans le jeu politique aurait permis de favoriser la transition démocratique en désamorçant les conflits auxquels elle aurait pu donner lieu ${ }^{6}$. Grâce aux larges compétences qui leur ont été reconnues, les cours constitutionnelles d'Europe centrale et orientale auraient réussi à « imprim[er] leur autorité sur le processus de transition constitutionnel $»^{7}$ et $~ "$ en partie, désamorc[er] certains des conflits les plus explosifs que ce soit sur des questions de fond ou au sujet des luttes entre pouvoirs constitués ${ }^{8}$. Ce faisant, elles auraient «largement contribué au processus de transition démocratique " ${ }^{9}$. Ces expériences ont d'ailleurs pu laisser croire, un temps, à la fin de l'histoire constitutionnelle ${ }^{10}$. Or, malgré les pouvoirs dévolus aux cours constitutionnelles d'Europe centrale et orientale pour conduire la «transition démocratique ", la conflictualité constitutionnelle n'a pas disparu. Ces dix dernières années, plusieurs cours constitutionnelles d'Europe centrale et orientale ont été confrontées à des majorités gouvernementales porteuses d'un projet politique qualifié d'autoritaire, d'illibéral, ou de populiste dont l'une des caractéristiques est la remise en cause de la place qu'occupent les cours constitutionnelles dans le système institutionnel. Les dynamiques constitutionnelles à l'œuvre depuis une dizaine d'années, notamment en Europe centrale et orientale, ont donc conduit la doctrine à repenser le rapport des cours constitutionnelles au « conflit».

3 La figure de la victime a ainsi pris le pas sur celle de l'arbitre pour caractériser la position du juge constitutionnel à l'égard de conflits constitutionnels contemporains. Les cours ont en effet eu à affronter, outre des discours hostiles ${ }^{11}$, les entreprises de majorités illibérales visant à ignorer ${ }^{12}$, contourner ${ }^{13}$ ou surmonter ${ }^{14}$ leurs décisions (decision reversal). Les cours ont également eu à faire face à des tentatives visant à réduire leur champ de compétences, complexifier leurs modalités de saisine ou paralyser leur organisation interne pour réduire leurs capacités d'action (courtstripping $)^{15}$. Les cours ont enfin eu à réagir aux démarches visant à modifier leur composition afin de parvenir à nommer une majorité des juges au sein de la Cour ${ }^{16}$ voire à exercer des pressions plus directes sur les juges afin qu'ils retiennent l'interprétation proposée ${ }^{17}$ (court-packing). Les auteurs axiologiquement engagés en faveur d'un modèle politique démo-libéral y ont vu la mise en œuvre d'une " politique $d u$ ressentiment» à l'encontre d'acteurs ayant fait preuve d'activisme pour imposer le modèle de l'État de droit ${ }^{18}$. Ils se sont ainsi attachés à dénoncer les « attaques » menées à l'encontre d' " une cible facile ${ }^{19}$ " (car dépourvue de légitimité élective et en proie à la difficulté contre-majoritaire) analysées comme autant de violations, si ce n'est de la Constitution elle-même, à tout le moins des valeurs fondatrices du constitutionnalisme ${ }^{20}$. À l'inverse, l'idée d'un juge victime des conflits a aussi alimenté un discours critique à l'égard de ce qu'il est convenu d'appeler la démocratie constitutionnelle; pointant les limites de la justice constitutionnelle comme mécanisme de garantie de la démocratie. Ce tigre de papier ne protégerait efficacement la démocratie qu'en l'absence de menace ${ }^{21}$. Arbitre ou victime, le juge constitutionnel ne semble pas devoir être considéré comme un protagoniste à part entière du conflit constitutionnel. Il apparait toujours maintenu dans une forme d'extériorité à l'égard de 
tels conflits auxquels il ne serait pas susceptible de prendre part ou de se soustraire, d'y perdre ou d'en tirer profit, de contribuer à sa résolution ou à sa prolongation

4 La figure du stratège est donc rarement mobilisée pour appréhender le rôle joué par les cours constitutionnelles lors de tels conflits ${ }^{22}$. Cela tient sans doute au fait que les définitions disponibles du conflit constitutionnel permettent difficilement d'envisager le pouvoir du juge constitutionnel ${ }^{23}$. Pour dépasser l'alternative réductrice entre omnipotence et impuissance des cours à l'égard du conflit constitutionnel, il importe donc de se doter d'une définition qui permette d'envisager le juge comme un acteur libre dans son activité d'interprétation des énoncés constitutionnels. Celui-ci se voit toutefois contraint par la configuration du système institutionnel au sein duquel il opère et par la capacité des autres acteurs, en particulier les autorités politiques, à proposer des interprétations concurrentes de la Constitution dont ils cherchent également à faire reconnaitre l'authenticité. En effet, loin d'être une activité monopolistique et pérenne, l'interprétation authentique de la Constitution, celle « ̀̀ laquelle l'ordre juridique fait produire des effets, qui ne peut être valablement contestée et qui s'incorpore par conséquent au texte interprété ", doit plutôt être appréhendée comme étant instable et concurrentielle ${ }^{24}$. Le conflit constitutionnel sera en conséquence considéré ici comme la situation dans laquelle un ou plusieurs organes constitutionnels s'oppose(nt) à une prétention à l'interprétation authentique de la Constitution émise par un autre, soit en niant son authenticité, soit en entreprenant de lui en substituer une autre. Il renvoie donc à des situations dans lesquelles «la question de la primauté d'un interprète constitutionnel sur un autre est ouvertement posée $»^{25}$. En effet, si l'interprétation authentique est celle qui produit des effets juridiques, son authenticité dépend, au moins dans une certaine mesure, de la réception qui en est faite par les autres acteurs du système. L'authenticité de l'interprétation peut donc elle-même être soumise à interprétation. Si, en outre, l'interprétation authentique est celle qui ne peut être valablement contestée car aucune autorité n'est habilitée à annuler la décision qui soutient cette interprétation, cela ne signifie pas pour autant qu'une autre interprétation authentique ne puisse pas lui être substituée.

5 Le pouvoir du juge constitutionnel d'émettre des interprétations constitutionnelles authentiques peut donc être "confronté à la résistance et au pouvoir de réplique des autres acteurs juridiques ${ }^{26}$. Confrontées à la remise en cause de leur pouvoir, les cours constitutionnelles "ne sont pas nécessairement dépourvues de toute marge de manœuvre ${ }^{27}$ et peuvent, elles aussi, opposer "un certain pouvoir de contre-réplique» aux organes concurrents ${ }^{28}$. Cette approche stratégique peut permettre de renouveler la manière d'appréhender les évolutions institutionnelles à l'œuvre actuellement dans certains États d'Europe centrale et orientale et le rôle que peuvent jouer les cours constitutionnelles dans un contexte illibéral. Elle permet de s'affranchir de présupposés axiologiques susceptible de fausser la perception à ce sujet. Il ne s'agira pas ici d'étudier les stratégies déployées par ces cours en tant que telles, ni de procéder à une étude de droit comparé29, mais plus modestement de montrer la possibilité d'une lecture stratégique de ces processus de transition au cours desquelles les cours constitutionnelles peuvent chercher à affirmer leur pouvoir pour résister (I) ou à le mettre au service de leurs concurrents pour s'adapter (II). 


\section{I] Les stratégies de résistance}

$6 \quad$ La résistance du juge constitutionnel aux entreprises des autorités politiques visant à remettre en cause ses prétentions à l'interprétation constitutionnelle authentique peut se manifester par l'adoption d'une stratégie de confrontation par laquelle il affirme un rapport de domination visant à faire reconnaitre sa primauté en tant qu'interprète la Constitution (A). La perception que la Cour se fait du rapport de force et des risques liés à la confrontation peut la conduire à tenter de minimiser le coût du conflit en optant pour une stratégie de conciliation (B).

\section{A/ La confrontation}

7 La confrontation vise pour la Cour à satisfaire totalement ses intérêts au détriment de son concurrent. Elle recouvre une dimension défensive et protectrice qui vise à annihiler les entreprises de l'organe concurrent ou à en minorer les effets (1) et une dimension offensive et préventive qui vise à leur dissuasion (2).

\section{La neutralisation}

8 Le pouvoir de sanction des actes législatifs ou réglementaires soumis à son contrôle constitue, sans nul doute, le principal moyen par lequel les Cours constitutionnelles peuvent s'opposer aux tentatives de remise en cause de leur pouvoir d'émettre des interprétations authentiques de la Constitution. Le juge constitutionnel peut ainsi s'opposer aux remises en causes législatives de son statut que les autorités politiques tentent de soustraire à son contrôle. C'est ainsi qu'en 2012, la Cour constitutionnelle roumaine a livré une interprétation « constructive» de l'article 146 de la Constitution qui dispose que la juridiction constitutionnelle « remplit d'autres attributions [que celles précédemment listées] prévues par la loi organique relative à la Cour». Sur ce fondement, le législateur organique avait habilité la Cour à contrôler la constitutionnalité des actes internes au Parlement en 2010. Or la majorité parlementaire entendait priver la Cour de cette compétence afin de l'empêcher de contrôler les actes par lesquels elle avait démis les présidents de chambre de leurs fonctions et avait modifié la loi organique à cette fin. La Cour, saisie de cette loi organique, a considéré qu'une forme d'" effetcliquet » devait jouer à son profit. Écartant l'idée d'un parallélisme des formes, la Cour affirme qu'en vertu de la Constitution, le législateur organique n'est compétent que pour conférer des compétences supplémentaires au juge constitutionnel, mais pas pour lui en retirer ${ }^{30}$. Le tribunal constitutionnel polonais s'est également opposé à une tentative de soustraire à son contrôle une loi modifiant son statut. Lorsqu'en décembre 2015, la majorité PIS a modifié la loi relative au tribunal constitutionnel pour paralyser son fonctionnement, elle a déclaré cette modification d'application immédiate. Autrement dit, le législateur soutenait que la loi devait être contrôlée suivant la procédure contentieuse qu'elle définissait elle-même. Cela aurait conduit à paralyser l'action du tribunal qui n'aurait pas pu rendre sa décision puisqu'il ne reconnaissait, à cette date, que douze juges valablement élus alors que la loi soumise à son contrôle fixait un quorum de 13 juges pour pouvoir se prononcer. Considérant que soumettre le Tribunal à un statut dont la constitutionnalité n'aurait pas été examinée serait contraire au principe d'un État démocratique de Droit, la juridiction constitutionnelle a 
refusé de reconnaitre l'applicabilité immédiate de la loi de décembre 2015 et a déclaré l'inconstitutionnalité de la plupart de ses dispositions ${ }^{31}$.

9 Le pouvoir de sanction des actes constitutionnel peut également offrir au juge constitutionnel, s'il parvient à se l'arroger, une ressource importante pour tenter de neutraliser les « lits de justice». C'est ce qu'illustre le cas de la Cour constitutionnelle hongroise. Invitée à contrôler la nouvelle constitution en 2011, la Cour a d'abord cherché à affirmer sa compétence en matière de contrôle des lois de révision constitutionnelle. Si elle rappelle que de 1989 à 2011, elle s'est toujours refusée à contrôler la constitutionnalité des révisions constitutionnelles, elle ouvre la voie à un contrôle de leur procédure d'adoption en précisant qu'une telle compétence " ne peut être exclue en matière de contrôle des dispositions de la Constitution quant à leur invalidité au regard du droit public $\|^{32}$. Sur le fondement de cette décision, la Cour a été amenée, le 28 décembre 2012, à invalider les «dispositions transitoires à la loi fondamentale " adoptées le 31 décembre 2011 en procédant à leur déconstitutionnalisation ${ }^{33}$. Alors même que la Constitution avait été révisée le 18 décembre 2012 pour prévoir l'incorporation de ces dispositions transitoires au sein de la loi fondamentale, la Cour relève une manœuvre visant à faire de ces dispositions transitoires « un acte se substituant à la loi fondamentale au lieu de l'amender ». Elle affirme sa compétence pour contrôler cet acte "qui ne fait pas partie de la loi fondamentale et qui ne peut être regardé comme une révision ou un amendement de la loi fondamentale $\aleph^{34}$. Le Constituant a réagi par l'adoption du quatrième amendement à la loi fondamentale. Cette fois, la Cour n'a pas cherché à annuler cette révision mais a procédé à l'interprétation neutralisante de certaines de ses dispositions. L'article 19 de cette loi de révision constitutionnelle modifiait les dispositions finales de la constitution en disposant que "les décisions de la Cour constitutionnelle antérieures à l'entrée en vigueur de la loi fondamentale [le 1er janvier 2012] sont annulées ", et ce, afin d'empêcher la Cour de se référer à son ancienne jurisprudence. Or le juge constitutionnel a interprété cette disposition de manière à en minorer la portée. Il a considéré qu'elles ne lui interdisaient pas de continuer à se référer à son ancienne jurisprudence, mais lui imposait seulement de justifier de manière détaillée les raisons pour lesquelles elle y recourait ${ }^{35}$.

Le pouvoir d'organisation interne de la Cour constitutionnelle peut également servir à neutraliser une attaque de la majorité gouvernementale. Investi de compétences spécifiques quant à l'organisation de la Cour, son Président peut ainsi être amené à jouer un rôle de premier plan. En Pologne, après que la Diète ait nommé cinq juges constitutionnels alors que le Tribunal lui avait enjoint de s'en abstenir jusqu'à ce qu'il se soit prononcé sur la constitutionnalité des nominations opérées sous la précédente législature, le Président du Tribunal a octroyé à ces cinq personnalités le statut d'agents du tribunal n'exerçant pas de fonction judiciaire. Il a ensuite admis à siéger deux des cinq juges ainsi élus après que le Tribunal a eu validé leurs nominations, mais a refusé, jusqu'à son départ, de permettre aux trois autres juges de siéger.

\section{La dissuasion}

Le pouvoir d'injonction, lorsqu'il est reconnu à la Cour constitutionnelle comme c'est le cas en Pologne, constitue un moyen de dissuasion privilégié. Alors que la Cour avait à statuer sur la régularité des nominations opérées par la majorité sortante en octobre 2015, la Cour décide, en vertu des articles 755(1) et 730(2) du Code de procédure civile, combinés à l'article 74 de la loi sur le tribunal, de prendre des mesures préventives 
visant à demander au Sejm de s'abstenir d'élire de nouveaux juges, jusqu'à ce qu'une décision définitive soit rendue. Toutefois, arguant d'une composition irrégulière de la Cour, la majorité gouvernementale refuse de tenir compte de cette injonction et procède à l'élection de cinq juges le 2 décembre 2015 soit la veille de la décision définitive du Tribunal qui validera l'élection de trois des cinq juges nommés par la majorité précédente.

recours aux obiter dictum peut être mobilisé par les juridictions constitutionnelles dépourvues d'un pouvoir d'injonction. Les Cours constitutionnelles roumaines et slovaques ont mobilisé cette technique pour dénoncer les comportements de la majorité gouvernementale voir la menacer. En 2011, la Cour constitutionnelle roumaine avait à examiner a priori et avec effet suspensif la loi qui la privait de sa compétence de contrôler les actes internes du Parlement. Sans attendre sa décision, le Gouvernement décide d'adopter une ordonnance d'urgence ayant le même contenu mais avec effet immédiat pour soustraire cette décision au contrôle de la Cour. À l'occasion de son contrôle de la loi initiale dont le contenu a été repris par l'ordonnance gouvernementale d'urgence, la Cour note qu'une telle manœuvre soulève pour débat " le comportement inconstitutionnel et abusif du gouvernement à l'égard de la Cour $\aleph^{36}$. Elle indique ainsi que la loi initiale étant inconstitutionnelle, l'ordonnance gouvernementale d'urgence ne peut en reprendre le contenu ${ }^{37}$. Finalement saisie de la loi ratifiant cette ordonnance gouvernementale d'urgence, la Cour l'a censurée ${ }^{38}$. La Cour constitutionnelle slovaque s'est quant à elle faite plus menaçante encore en 2016. Le refus du Président de la République de nommer suffisamment de juges sur une liste établie par le Parlement et comprenant deux fois plus de candidats que de postes à pourvoir est jugé inconstitutionnel par la Cour qui indique que la non-application de la décision de la Cour par le Président serait « une violation de son serment constitutionnel et une violation intentionnelle de la Constitution qui devrait conduire à la mise en jeu de sa responsabilité par le Parlement». La Cour relève toutefois qu'elle est impuissante à assurer par elle-même l'exécution de ses décisions ${ }^{39}$.

13 Ces stratégies de confrontation par lesquelles les Cours tentent d'affirmer un rapport et de maintenir leurs positions peuvent leur servir à se présenter dans les conditions les plus favorables possibles au moment de mettre en œuvre une stratégie de négociation afin de minimiser les concessions à effectuer pour régler le conflit.

\section{B/ La conciliation}

14 La conciliation vise la satisfaction partielle des intérêts de la Cour que ce soit par la recherche d'une solution directement négociée avec l'organe constitutionnel concurrent (1) ou par la recherche d'un arbitre qu'elle sollicite (2).

\section{La négociation}

Un dialogue interorganique peut servir de cadre à une négociation formalisée. En Slovaquie, le Président de la Cour Ivan Fiačan a ainsi joué un rôle prépondérant pour permettre la nomination de nouveaux juges alors que la vacance de nombreux postes menaçait le fonctionnement de la Cour. C'est ainsi qu'il a pu, en mobilisant pour la première fois le pouvoir qu'il tient de l'article 20 paragraphe 1 du Règlement de la Chambre, intervenir devant les parlementaires. Il a ainsi pu prononcer un discours, 
sous forme de plaidoyer ${ }^{40}$, afin que le Parlement s'accorde sur une liste de candidats ce qu'il a finalement fait ${ }^{41}$.

Un compromis implicite peut également être envisagé. En témoigne la consécration, par le quatrième amendement à la loi fondamentale hongroise de la compétence de la Cour en matière de contrôle de la procédure d'adoption des lois de révision constitutionnelle sur saisine du Président de la République ${ }^{42}$. Cette disposition a pu être interprétée comme la matérialisation d'une volonté de la majorité gouvernementale de cantonner le contrôle des lois de révision à sa dimension procédurale après que la Cour ait manifesté quelques velléités quant à l'exercice d'un contrôle au fond ${ }^{43}$. On peut également y voir une forme de compromis, proposée à la Cour par le constituant et entérinée à l'occasion du contrôle de constitutionnalité de ce quatrième amendement. La Cour a tout d'abord souligné qu'elle ne pouvait contrôler les lois constitutionnelles que sur habilitation expresse du constituant. Elle a ensuite indiqué que l'exception tirée de «l'invalidité au regard du droit public » ne pouvait s'entendre que d'un strict point de vue procédural limité à la procédure d'adoption et de promulgation. Le hautcommissaire aux droits de l'Homme avait saisi la Cour en alléguant que l'accumulation des lits de justice opérée par les révisions successives de la Constitution entre 2011 et 2013 avait conduit à faire coexister des dispositions contradictoires au sein de la Constitution. La Cour a considéré qu'il s'agissait d'une question de fond et a refusé de se prononcer ${ }^{44}$. La Cour constitutionnelle slovaque n'a, quant à elle, pas réussi à faire entériner un compromis similaire ${ }^{45}$.

\section{La sollicitation}

Saisir un tiers indépendant est une ressource dont disposent les membres de la Cour lorsque l'action de la majorité gouvernementale vise à empêcher son entrée en fonction, à précipiter la fin de son mandat, ou encore à faire pression sur lui afin qu'il se prononce dans le sens souhaité par l'autorité politique impliquée dans le conflit. C'est ainsi qu'une juge à la Cour constitutionnelle roumaine a porté plainte en 2012 face aux menaces de mort dont elle aurait été l'objet. Le Président de la Cour avait quant à lui écrit aux institutions européennes afin de les alerter concernant « les attaques lancées par le Gouvernement et d'autres autorités publiques à l'encontre de la Cour et de ses juges ». En réponse, la commissaire européenne à la justice et le Président de la commission de Venise avaient appelé le Gouvernement à protéger l'indépendance de la Justice ${ }^{46}$. En Pologne également, les juges constitutionnels écartés ont intenté des actions devant les juridictions de droit commun, mais le Tribunal constitutionnel polonais nouvellement composé a nié leur compétence pour contrôler la régularité de la nomination des juges constitutionnels ${ }^{47}$.

Alerter l'opinion publique constitue le dernier recours à disposition des juges constitutionnels. Ainsi, lorsque, comme en Pologne, un acteur constitutionnel parvient à modifier la composition de la Cour et à nommer lui-même une majorité de juges, les " anciens " juges, mis en minorité, ne disposent plus que du pouvoir de publier des opinions séparées pour maintenir une forme de concurrence interprétative. Via des opinions dissidentes retransmises sur le site de la Cour, les juges minoritaires ont mis en doute la validité des décisions du Tribunal au regard de sa composition ${ }^{48}$. Mais les juges majoritaires ont réagi en modifiant le Règlement intérieur de la Cour pour circonscrire l'usage des opinions dissidentes à la discussion du dispositif ou des motifs de la décision et exclure la phase préparatoire au jugement ${ }^{49}$. 
19 Les stratégies de résistance ici reconstruites ont, pour la plupart, été mises en œuvre par les cours constitutionnelles immédiatement après la survenance des conflits et ont permis, dans un certain nombre de cas, leurs résolutions. Lorsque le conflit constitutionnel se prolonge, le rapport de force tend à évoluer en défaveur des cours, surtout si l'autorité politique rivale parvient à confirmer sa légitimité par les urnes, comme ce fut singulièrement le cas en Hongrie et en Pologne. Ces deux exemples permettent d'illustrer les stratégies d'allégeance que les cours constitutionnelles sont susceptibles de déployer pour satisfaire les intérêts de l'autorité politique concurrente tout en préservant leur pouvoir d'émettre des interprétations constitutionnelles authentiques.

\section{II] Les stratégies d'allégeance}

20 L'allégeance renvoie ici aux stratégies déployées par les Cours dans des situations où elles perçoivent en leur défaveur, le rapport de pouvoir qui les oppose aux autorités politiques. La renonciation vise, pour une Cour, à minimiser les pertes induites par cette situation en restreignant l'exercice de son pouvoir afin de mieux le conserver (A). La collaboration vise quant à elle à optimiser les gains en mettant son pouvoir au service de l'autorité politique à laquelle elle est en situation de conflit (B).

\section{A/ La renonciation}

La renonciation vise la satisfaction des intérêts de la partie concurrente au détriment de ceux de la Cour par anticipation d'une éventuelle réplique. Cette stratégie, qui vise à " s'affirmer sans confrontation frontale avec les pouvoirs politiques " ${ }^{50}$, peut se manifester par le fait que la Cour repousse sa prise de décision (1) ou relâche son contrôle (2).

\section{La temporisation}

Repousser la prise de décision peut être un premier moyen à disposition des cours pour esquiver un conflit interprétatif avec la majorité gouvernementale ${ }^{51}$. Repousser la prise de décision évite de se prononcer dans la foulée de l'adoption de la loi. En décidant de ne pas décider, le juge peut ainsi manifester une certaine indépendance à l'égard de la majorité sans pour autant freiner cette dernière. S'il prononce une inconstitutionnalité plus tard, celle-ci apparaitra moins coûteuse au législateur puisque l'attention sera moindre et/ou qu'elle aura déjà produit les effets qui étaient attendus d'elle. Après le référendum organisé le 29 juillet 2012, la Cour constitutionnelle roumaine n'est pas parvenue à dégager une majorité pour adopter une décision concernant la validation ou l'invalidation des résultats du référendum et a retardé sa décision de plusieurs mois $^{52}$. La Cour constitutionnelle de République tchèque a quant à elle refusé en 2013 la mise en accusation du Président de la République au motif que son mandat avait expiré53 $^{5}$. Le cas le plus emblématique est sans doute celui de la Cour constitutionnelle hongroise saisie de la loi sur l'enseignement supérieur (qui visait la CEU financée par Soros considéré comme un des principaux opposants au régime) au printemps 2017. Celle-ci a d'abord eu recours à des procédures exceptionnelles pour retarder la décision particulièrement sensible politiquement : la création d'un comité ad hoc, composé de greffiers chargés de préparer la procédure décisionnelle et des demandes de clarifications adressées aux plaignants et à différentes institutions étatiques ${ }^{54}$. En 
décembre 2018, la Cour constitutionnelle hongroise a également déclaré l'inconstitutionnalité d'une loi relative à la propagande électorale adoptée en juin 2017 soit plus de six mois après les élections législatives ${ }^{55}$. En Pologne, le Tribunal constitutionnel a quant à lui prononcé l'inconstitutionnalité d'une loi mémorielle plus d'un an après qu'elle lui ait été soumise par le Président de la République et après que le Gouvernement ait retiré l'un de ses articles les plus controversés ${ }^{56}$. La récente décision du tribunal constitutionnel polonais qui conclut à l'inconstitutionnalité de certaines dispositions des traités européens a elle aussi été rendue après quatre reports consécutifs ${ }^{57}$.

Surseoir à statuer dans l'attente de la décision d'un autre acteur juridique peut servir un but identique. Dans la décision de la cour constitutionnelle hongroise relative à la loi sur l'enseignement supérieur précité, la Cour avait de surcroit, en juin 2018, suspendu la procédure se déclarant obligée d'attendre la décision de la Cour de justice de l'Union quant au recours en manquement engagé contre la Hongrie. Elle a procédé de la même manière au sujet de la loi sur la transparence des organisations financées depuis l'étranger ${ }^{58}$, en attendant les résultats de la procédure d'infraction lancée par l'Union européenne ${ }^{59}$.

\section{Le retrait}

Réduire le nombre d'inconstitutionnalités constitue le moyen le plus évident pour éviter au juge un conflit avec la majorité gouvernementale. Cela peut d'abord passer par un refus de décider de la Cour. La Cour peut d'abord restreindre l'accès à son prétoire en refusant par exemple de reconnaitre l'intérêt à agir du requérant. La cour constitutionnelle hongroise a eu recours à cette technique s'agissant d'une requête dirigée contre la loi sur les procédures de vote qui excluait du vote par correspondance les citoyens domiciliés en Hongrie mais résidant à l'étranger le jour de l'élection. La Cour a jugé la requête irrecevable en considérant que le requérant n'était pas personnellement affecté par la règle dès lors... qu'il était domicilié en Hongrie ${ }^{60}$. Un moyen tout aussi radical et coûteux est celui qui consiste à décliner sa compétence à l'instar du Tribunal constitutionnel polonais. Ce dernier a refusé d'invalider la nomination de la présidente de la Cour suprême opérée par la précédente majorité et de mettre fin à son mandat. Il s'est déclaré incompétent pour contrôler l'acte final de nomination adopté par le Président de la République dans le cadre de ses attributions constitutionnelles $^{61}$. Les cours peuvent également se refuser à censurer une irrégularité constitutionnelle qu'elles constatent au motif qu'elle n'est pas suffisamment substantielle pour justifier une censure comme l'a fait la Cour constitutionnelle hongroise au sujet d'une méconnaissance d'une disposition du règlement de l'Assemblée ${ }^{62}$. Adopter une attitude de déférence, respectueuse de la compétence du législateur peut permettre à une cour constitutionnelle d'éviter un conflit avec la majorité gouvernementale sans pour autant renoncer à contrôler son action. Les cours peuvent ainsi recourir à l'interprétation conforme et aux réserves d'interprétation afin d'éviter la censure de la loi tout en imposant leur propre interprétation de la Constitution. La Cour constitutionnelle hongroise semble utiliser cette technique lorsqu'une loi particulièrement sensible politiquement est en jeu comme la loi dite " Stop Soros » qui crée une infraction d'aide à l'immigration illégale dont la portée a été restreinte aux seuls discours incitant à l'immigration illégale ${ }^{63}$. Toutefois, les cours ne peuvent pas éviter toute forme de censure sous peine de nuire à terme à leur 
attractivité si les requérants jugent leur cause perdue d'avance. Elle peut cependant s'attacher à réduire le coût politique d'une censure.

Réduire le coût d'une inconstitutionnalité: Une autre méthode consiste à privilégier une censure fondée sur une inconstitutionnalité formelle plutôt que substantielle afin de réduire le coût de la censure pour le gouvernement. Cela permet de ne pas remettre en cause le choix politique de la majorité et d'éviter que la décision d'inconstitutionnalité n'apparaisse comme un désaveu ou la prétention à imposer une interprétation constitutionnelle concurrente. Il s'agit alors de minimiser le risque de réaction à une censure. La Cour constitutionnelle hongroise a ainsi choisi de censurer la loi créant une cour administrative suprême pour méconnaissance de la compétence du législateur cardinal (organique) plutôt que de se prononcer au regard du principe d'indépendance de la justice ${ }^{64}$. La Cour hongroise a également multiplié les censures pour omission législative en mentionnant parfois explicitement son intention de "sauver " la législation et qui laissent la majorité gouvernementale libre de remédier ou non à l'omission constatée ${ }^{65}$.

La stratégie de renonciation, essentiellement passive, consiste donc essentiellement pour une cour constitutionnelle, à ne pas faire usage de son pouvoir à l'encontre de l'autorité politique qui le concurrence dans son activité d'interprétation de la constitution. Une stratégie plus active peut être identifiée lorsqu'une cour met son pouvoir au service de son concurrent ce qui lui permet d'exercer son pouvoir sans risquer une réplique de sa part.

\section{B/ La collaboration}

La collaboration renvoie aux stratégies par lesquelles les cours tentent de satisfaire à la fois leurs intérêts et ceux de l'autorité politique concurrente. Les cours peuvent d'abord utiliser le contrôle de constitutionnalité comme un mécanisme permettant de justifier les décisions émises par les autorités politiques et les propositions d'interprétation constitutionnelle qu'elles véhiculent (1). Elles peuvent mobiliser cette compétence pour établir une alliance contre des interprètes concurrents (2).

\section{La justification}

Susciter l'intervention d'une autorité politique peut être un premier moyen de collaboration. Plusieurs décisions du tribunal constitutionnel reconnaissant la compétence du législateur en matière d'organisation du pouvoir judiciaire ont ouvert la voie aux réformes gouvernementales remettant en cause l'indépendance du pouvoir judiciaire ${ }^{66}$. Dès 2017, le contrôle de constitutionnalité exercé par le tribunal constitutionnel polonais a permis au législateur d'intervenir Conseil supérieur de la Magistrature ${ }^{67}$. Le Tribunal, via un obiter dictum, précise que les nominations sont faites au sein des juridictions, mais pas nécessairement par les juges et que le législateur bénéficie d'une compétence discrétionnaire pour régler cette question ce qu'il n'a pas manqué de faire en confiant à l'Assemblée la compétence de nomination sans possibilité de recours contre la procédure de nomination. Il en a été de même au sujet de la nomination du Président de la Cour suprême. Le tribunal a jugé que cette procédure ne pouvait pas être réglée par le Règlement intérieur de la Cour et relevait de la compétence du législateur qui est donc intervenu pour réviser la loi sur la Cour suprême $^{68}$. La Cour constitutionnelle peut ainsi servir ses propres intérêts (en exerçant 
un contrôle susceptible d'aboutir à une sanction) et ceux de la majorité gouvernementale (en fondant sa compétence et sa légitimité à intervenir).

Soutenir l'intervention peut également être un moyen de faire preuve de déférence à l'égard d'une autorité politique et plus précisément de la majorité gouvernementale. Outre les «brevets de constitutionnalité » qu'elles sont susceptibles de décerner, les cours peuvent déployer une politique de soutien plus direct. C'est ainsi que la cour constitutionnelle hongroise a pu apparaitre "comme un renfort opportun de la politique gouvernementale $"{ }^{69}$ à la suite de sa décision consacrant l'identité constitutionnelle comme limite à la compétence de l'Union européenne ${ }^{70}$. Cette dernière est en effet intervenue après un double échec du gouvernement dans sa tentative de légitimer, par le suffrage, son opposition à la politique européenne de relocalisation des demandeurs d'asile. Le référendum qu'il avait initié sur le sujet n'avait pas atteint le quorum de participation nécessaire et la révision constitutionnelle, engagée dans la foulée, afin de rendre inconstitutionnels les quotas européens de relocalisation avait été rejetée à deux voix près ${ }^{71}$. La communication institutionnelle des cours peut également être mise au service de la majorité gouvernementale. En Pologne, la présidente de la Cour a pu apporter activement son soutien à la politique de la majorité en défendant, lors d'un entretien sur une chaine de télévision gouvernementale, la constitutionnalité des projets de loi de réforme judiciaire affirmant qu'ils ne constituaient pas « une menace pour la séparation des pouvoirs » et rencontraient « les attentes de la société $»^{72}$.

\section{La protection}

Assujettir les juges nationaux à la norme constitutionnelle telle que proposé par les interprètes politiques et authentifié par les cours constitutionnelles peut être un objectif commun aux deux institutions. Les cours constitutionnelles, contraintes de minorer le contrôle qu'elles exercent sur les actes adoptés par les autorités politiques, peuvent ainsi s'adapter en réorientant leur contrôle vers les actes juridictionnels. La nouvelle constitution hongroise adoptée en 2011 a largement redessiné les compétences de la Cour constitutionnelle. Un nouveau recours contre les décisions juridictionnelles a ainsi été consacré et lui a permis d'augmenter le nombre de décisions rendues annuellement ${ }^{73}$. Elle peut, par ce biais, prononcer l'inconstitutionnalité de décisions juridictionnelles susceptibles d'entraver l'action du gouvernement. Un exemple topique peut être trouvé dans une décision rendue en matière de contentieux électoral. La Cour constitutionnelle a censuré une décision de la Cour suprême à la suite d'une plainte d'un candidat du Fidesz sanctionné pour son affiche électorale qui assimilait le parti et le gouvernement ${ }^{74}$. Même en l'absence d'une telle compétence, une Cour, via le contrôle de constitutionnalité, peut chercher à minorer la concurrence interprétative que les juridictions ordinaires sont susceptibles d'opposer à la majorité gouvernementale. Le contrôle par le tribunal constitutionnel polonais des lois relatives à l'organisation judiciaire en témoigne. Dès 2017, le contrôle de constitutionnalité exercé par le tribunal constitutionnel polonais a ouvert la voie à une refonte du Conseil supérieur de la Magistrature ${ }^{75}$. Il a par la suite dénié toute compétence aux juridictions ordinaires pour connaitre d'un recours contre la procédure de nomination des juges constitutionnels protégeant ainsi le Gouvernement (et certains juges constitutionnels) contre une remise en cause des procédures de nominations litigieuses de décembre $2015^{76}$. Il a également interprété les dispositions relatives au droit de grâce du Président comme lui permettant d'en faire usage avant 
même que l'accusé ait fait l'objet d'une condamnation en justice ${ }^{77}$. Enfin, la loi qui permettait jusqu'ici au Défenseur des droits de rester en poste faute d'accord pour désigner son successeur a récemment été jugée inconstitutionnelle ${ }^{78}$. Le juge constitutionnel peut également soutenir la majorité gouvernementale en arbitrant favorablement les litiges de compétences qui l'opposent au pouvoir judiciaire. Le 21 avril 2020, le tribunal constitutionnel polonais a ainsi considéré que la Cour suprême n'était pas compétente pour adopter une résolution dénonçant l'absence d'indépendance de sa propre chambre spéciale chargée de la discipline des juges dont les membres avaient été nommés par le KRS (équivalent du Conseil de la magistrature passé sous le contrôle du Gouvernement) ${ }^{79}$.

31 Affronter les juges supranationaux : Les cours constitutionnelles peuvent également défendre les interprétations constitutionnelles émises par la majorité gouvernementale contre de possibles remises en causes par les juridictions européennes. La CJUE et la CEDH, soucieuses d'assurer la primauté de leurs ordres juridiques respectifs sur le droit (y compris constitutionnel) des États membres sont donc amenés eux aussi, à interpréter les actes constitutionnels nationaux notamment lorsqu'ils doivent déterminer les compétences respectives de l'Union et des États membres. La Cour peut ainsi mettre son pouvoir d'interprétation authentique de la Constitution comme un instrument au service des politiques gouvernementales d'opposition à l'Union européenne. Le mouvement de remise en cause de la primauté du droit de l'Union par les cours constitutionnelles européennes fait l'objet d'une abondante littérature et on se contentera ici de mentionner deux des exemples les plus marquants. Le premier repose sur l'idée d'une atteinte substantielle de l'Union à l'identité constitutionnelle nationale. En 2016, la Cour constitutionnelle hongroise s'est ainsi reconnue compétente pour contrôler l'exercice par l'Union européenne de ses compétences non seulement au regard des droits fondamentaux mais également au regard des principes de souveraineté et d'identité constitutionnelle. Elle semble conférer à cette dernière notion une valeur supraconstitutionnelle en affirmant qu'elle est «une valeur fondamentale » qui n'est pas créée par la loi fondamentale mais simplement reconnue par elle et qui ne peut être aliénée par la voie d'un traité international ${ }^{80}$. Cette solution a été consacrée en 2018 par le septième amendement qui opère une codification de la notion d'identité constitutionnelle dans la loi fondamentale. L'article $\mathrm{R}$ al. 4 dispose que tous les organes de l'État ont l'obligation de protéger l'identité constitutionnelle et la culture chrétienne de la Hongrie. Le second exemple repose quant à lui sur l'idée d'une méconnaissance formelle par l'Union des compétences conservées par l'État puisqu'il s'agit de la récente et retentissante décision du tribunal constitutionnel polonais par laquelle il a jugé pour la première fois certains articles du Traité de l'Union européenne incompatible avec la Constitution. Il a ainsi jugé, à la demande du Premier ministre, que l'Union européenne imposait à la République de Pologne des obligations qui dépassent les compétences que cette dernière lui a conférées par traité ${ }^{81}$. Les deux premiers alinéas de l'article $1^{\mathrm{er}} \mathrm{du}$ TUE, combinés à l'article 4 (qui pose le principe de coopération loyale), sont considérés comme la source d'obligations ultra vires imposées à la République polonaise qui font perdre à sa Constitution son caractère de loi suprême et à l'État sa souveraineté. L'article 19, qui dispose que les États membres établissent les voies de recours nécessaires pour assurer une protection juridictionnelle effective dans les domaines couverts par le droit de l'Union, fait lui aussi l'objet d'une déclaration d'inconstitutionnalité. La primauté du droit de l'Union et le rôle des juges nationaux en tant que juge de droit commun de l'Union sont ainsi frontalement remis en cause. Les 
juridictions polonaises se voient ainsi privées de la possibilité d'agir sur le fondement de l'article $19 \S 1$ TUE pour contrer les attaques du pouvoir en place contre l'indépendance de la justice. Dans les deux cas, le pouvoir d'interprétation authentique de la Constitution délivrée par les cours vient donc au soutien du Gouvernement dans son opposition aux institutions européennes.

Les stratégies de collaboration actuellement déployées par les cours constitutionnelles hongroise et polonaise laissent donc à penser que de nouvelles figures, celles de l'allié, du complice, ou encore de l'affidé, risque de s'imposer pour caractériser le rôle que joue le juge constitutionnel dans les processus d'illibéralisation des régimes constitutionnels.

\section{NOTES}

1. Voir les entrées «conflit » et «stratégie » du Trésor de la langue française disponible sur le site du CNTRL.

2. Dans le discours doctrinal français à tout le moins. V. cependant et entre autres : Meunier JACQUES, Le pouvoir du Conseil Constitutionnel : essai d'analyse stratégique, Paris, LGDJ, 1994 ; Michel TROPER et al (dir.), Théorie des contraintes juridiques, Paris, Bruylant/LGDJ, « La pensée juridique », 2005 ; Guillaume TUSSEAU, « Le Gouvernement [contraint] des juges. Les juges constitutionnels face au pouvoir de réplique des autres acteurs juridiques - ou l'art partagé de ne pas pouvoir avoir toujours raison » Droits, 2012, vol. 1, n 55, p. 41-84 ; Eric MILLARD, « Les contraintes : entre ressources stratégiques et théories de la régularité », Droits, $n^{\circ} 55,2012$, p. 23-40.

3. V. en ce sens: Lee EPSTEIN, Jack KNIGHT \& Olga SHVETSOVA, "The rôle of constitutional courts in the establishment and maintenance of demoratic systems of governement » Law \& Society Review, vol. 35, $\mathrm{n}^{\circ} 1,2001$, pp. 117-164.

4. C'est ainsi que pour Alec STONE SWEET, «Une cour constitutionnelle remplit plusieurs fonctions qui facilitent la transition vers la démocratie [en fournissant] un système de résolution pacifique des conflits à ceux qui ont contracté un nouveau départ, à la lumière d'un système autoritaire et violent [...], un mécanisme pour purger les lois de leurs aspects autoritaires, étant donné que la nouvelle législature risque d'être surchargée de travail [...] et le vecteur d'une nouvelle rhétorique de la légitimité étatique fondée sur le respect des valeurs et des droits démocratiques et le rejet de la rhétorique antérieure " Alex SWEET STONE, "Constitutional courts " in Michel ROSENFELD et Andras SAJo (eds), The Oxford Handbook of comparative constitutional law, Oxford, Oxford University Press, 2012, Chapitre 38, p. 8.

5. Kim Lane SCHEPPELE, "Political contextst of judicial activism in post-soviet europe " in Saïd Amir ARJOMAND (ed.), Constitutionalism an Political reconstruction, Leiden, Brill, International Comparative Social Studies, 2007, p. 315-336 (cit. p. 316). Plus généralement, sur le contrôle de constitutionnalité dans les États d'Europe de l'Est voir: Wojciech SADURSKI, Rights before courts, A study of constitutional courts in postcommunist states of central and eastern Europe, Dordrecht, Springer, 2nde éd., 2014.

6. V. par ex. : Wojciech SADURSKI, « Postcommunist constitutionnal courts in search of political legitimacy ", Florence, Institut universitaire européen, 2001, p.1: "l'ensemble des pays postcommunistes d'Europe centrale et orientale ont une cour constitutionnelle, et si l'efficacité de ces tribunaux varie, tous, sans exception ont imprimé leur autorité sur le processus de transition 
constitutionnelle». V. également: Jonathan BOND, « Concerning constitutional courts in eastern Europe ", International Public Policy Review, vol. 2, n² 2, 2006, p. 6.

7. Wojciech SADURSKI, «Postcommunist constitutionnal courts in search of political legitimacy ", EUI Law, 2011, n 11, p. 1.

8. Ibid.

9. Jonathan BOND, «Concerning constitutional courts in eastern Europe », International Public Policy Review, 2006, vol. 2, n², p. 6.

10. Certains auteurs se sont même interrogés sur le point de savoir « si la promotion de la justice constitutionnelle ne s'accompagne pas d'une stérilisation juridictionnelle qui rend désormais impossible la survenance de tout véritable conflit constitutionnel, ce dernier étant condamné à n'être plus qu'un désaccord herméneutique sur les dispositions constitutionnelles résolu par le juge ». V. Jacky HUMMEL, " Les conflits constitutionnels. Définition(s) d'un objet d'étude » in Jacky HUMmel (dir.), Les conflits constitutionnels, Rennes, PUR, 2010, p. 16.

11. Ces entreprises se sont traduites par un discours officiel dénonçant le manque d'indépendance et la politisation des cours, les irrégularités procédurales voire les violations de la Constitution dont elles se seraient rendues coupables. En 2011, József sZÁJERN, dirigeant du FIDESZ et rédacteur de la loi nouvelle loi fondamentale hongroise déclarait ainsi que : « le contrôle de constitutionnalité est un contrepouvoir politique dans toutes ses dimensions " (cité par Petra Léa LÁNCos, "Passivist Strategies Available to the Hungarian Constitutional Court », ZaöRV , 2019, $\mathrm{n}^{\circ}$ 79, p. 971-993). En 2015, le ministre de la justice polonais niait quant à lui la validité d'une décision du Tribunal constitutionnel en déclarant que «dans ce cas particulier, heureusement, nous n'avons pas affaire à un jugement au terme d'une procédure légale. Cette réunion de juge au sein de la Cour n'est pas la réunion de la Cour constitutionnelle mais une réunion de juge qui ont sans succès essayé de rendre un jugement pour lequel ils n'avaient pas compétence puisqu'ils ont agi en violation de la loi sur la Cour constitutionnelle qui gouverne son fonctionnement et en violation de la Constitution" (cité par Alexandra KUSTRA, «Poland's constitutional crisis. From court-packing agenda to denial of Constitutional Court's judgments », Toruńskie Studia Polsko-Włoskie-Studi Polacco Italiani di Toruń, 2016, vol. 12, p. 343-366).

12. Ce refus de reconnaitre les décisions de la cour a pu se traduire par la mise en cause de l'impartialité ou de la qualité de ses membres afin qu'ils se déportent, soient récusés ou fassent l'objet de poursuites disciplinaires. En 2016, le Président de la République slovaque a déposé une requête devant la Cour constitutionnelle afin que le jugement soit annulé en raison de la partialité d'un juge constitutionnel et qu'une procédure disciplinaire soit mise en œuvre à son encontre (V. Commission de Venise, Opinion $n^{\circ}$ 877/2017. CDL Ref (2017)013. Slovak republic decisions by the constitutional court and further relevant texts). En 2017, le procureur général de la République de Pologne a quant à lui déposé une requête pour que certains juges constitutionnels nommés suivant une procédure contestée par la majorité soient suspendus et obtenu satisfaction grâce au soutien de la présidente du Tribunal nouvellement nommée par la majorité. Cela peut également passer par le refus du gouvernement de procéder à la publication des décisions du Tribunal constitutionnel au motif de sa composition irrégulière comme ce fut le cas en Pologne au sujet des décisions du Tribunal Constitutionnel rendues en 2016 par lesquels il concluait à l'inconstitutionnalité de lois organisant le blocage de son fonctionnement.

13. Les acteurs constitutionnels peuvent à cette fin réitérer une décision annulée par le juge constitutionnel en tentant de la soustraire à son contrôle En Roumanie, en 2012, le Gouvernement a adopté deux ordonnances d'urgence, l'une visant à modifier les règles de quorum pour les référendums révocatoires, l'autre visant à retirer à la Cour sa compétence de contrôler la constitutionnalité des actes internes au Parlement alors même que deux lois ayant le même contenu était en cours d'examen par le juge constitutionnel (Elena-Simina TĂNĂSESCU, « Roumanie », AIJC 2012, 2013, p. 912 et s.). 
14. En s'appuyant sur une large coalition parlementaire FIDESZ-KDNP qui disposait à elle seule d'une majorité suffisante, le Gouvernement de Victor Orban a fait adopter une nouvelle Constitution en avril 2011 dont certaines dispositions visaient déjà à renverser la jurisprudence de la Cour. Cette dernière va l'année suivante déclarer l'inconstitutionnalité des dispositions transitoires à la loi fondamentale. En réaction le Parlement, agissant en tant que pouvoir constituant, va adopter le quatrième amendement à la loi fondamentale de Hongrie pour surmonter notamment certaines décisions rendues par la Cour constitutionnelle. Il a ainsi codifié au sein même de l'acte constituant un certain nombre de dispositions jugées inconstitutionnelles par la Cour quelques temps plus tôt (V. Commission de Venise, Avis 720/2013, CDL AD(2013)12, Avis sur le quatrième amendement à la loi fondamentale de Hongrie : «L'article L., par. 1, de la Loi fondamentale concerne la décision 43/2012 (relations familiales) ; l'article VII, la décision 1/2013 (reconnaissance des Églises) ; L'article IX, par. 3, la décision 1/2013 (limitation de l'accès aux médias des partis politiques) ; l'article IX, par. 5, les décisions 30/1992, 18/2004 et 95/2008 (liberté d'expression); l'article X, par. 3, la décision 69/2009 (autonomie des universités); l'article XI, par. 3, la décision 32/2012 (bourses d'étude) ; l'article XXII, par. 3, la décision 38/2012 (sans-abri) ».).

15. En Pologne, la majorité arrivée au pouvoir en novembre 2015 a immédiatement fait adopter une modification de la loi relative au tribunal constitutionnel (pour permettre notamment à la majorité nouvelle de nommer cinq nouveaux juges constitutionnels sans attendre la décision du juge constitutionnel quant à la régularité des nominations effectuées par la majorité sortante). Cette loi va être déclarée inconstitutionnelle presqu'intégralement. La majorité va toutefois adopter une nouvelle modification de la loi cardinale relative au tribunal constitutionnel. Entre autres mesures, elle prévoyait que le tribunal examine les saisines dans l'ordre de leur enregistrement, essentiellement en formation plénière dont le quorum était augmenté de 9 à 13 juges (alors qu'à cette date, seule 12 juges régulièrement nommés était en mesure de siéger) et en statuant à la majorité des deux tiers. Surtout, elle prévoit son applicabilité immédiate, la majorité alléguant que le contrôle de constitutionnalité de cette loi doit se faire selon les modalités qu'elle-même fixe (par ailleurs, le 30 janvier 2016, le Sejm adoptait le projet de loi relative au budget de l'État pour l'année 2016, qui réduisait d'environ de $10 \%$ le budget alloué à la Cour).

16. Les pouvoirs constitués peuvent ainsi chercher à agir sur la composition de la Cour afin d'y placer des juges qu'ils espèrent plus conciliants. En Hongrie, l'adoption de la loi fondamentale en 2011 a permis d'augmenter le nombre de juge constitutionnel passant de onze à quinze et en réformant la procédure de nomination pour permettre à la majorité de se dispenser d'une négociation avec les groupes parlementaires d'opposition. La nomination conséquente de nouveaux juges constitutionnels soutenus par le Fidesz a permis de modifier les équilibres au sein de la Cour dans un sens plus favorable à la majorité gouvernementale (V. Zoltán SZENTE, "The Political Orientation of Constitutional Judges in Hungary ", Constitutional Studies, 2016, vol. 1, n 1, p. 123-149). En Slovaquie, c'est au contraire la diminution du nombre de juge, provoquée par le refus du Président de la République de choisir suffisamment de candidats au sein de la liste proposée par la majorité gouvernementale qui, à compter de 2014 a menacé le fonctionnement de la Cour. En Pologne, juste avant le retour au pouvoir du PIS, victorieux des élections législatives d'octobre 2015, l'ancienne majorité, lors de sa dernière séance du 8 octobre 2015, avait procédé à la nomination de cinq juges constitutionnels : trois pour remplacer ceux dont le mandat arrivait à échéance le 6 novembre 2015 sous la VIIe législature, et deux dont pour remplacer ceux le mandat arrivait à échéance seulement en décembre, soit sous la VIIIe législature qui débutait le 12 novembre 2015. La majorité sortante a procédé de la sorte sur le fondement de la loi sur le tribunal constitutionnel modifié en juin 2015 afin de permettre ces cinq nominations. Fin octobre 2015, sans attendre la décision du juge constitutionnel, la nouvelle majorité, jugeant l'ensemble de ces nominations irrégulières (les deux opérées pour remplacer les juges dont le mandat expirait sous la VIIIème législature mais aussi les trois opérées pour 
remplacer les juges dont le mandat expirait sous la VIIème législature) les a annulées rétroactivement et elle a procédé à l'élection de cinq nouveaux juges.

17. En 2012, des juges constitutionnels roumains ont ainsi indiqué avoir subis différentes pressions (y compris des menaces de mort) alors qu'ils devaient se prononcer sur la validité du référendum relatif à la destitution du Président de la République (V. «Commission concerned by 'death threat' to Romanian judges », Euractiv, 9 aout 2012). En 2019 certains juges constitutionnels tchèques ont affirmé que le Président avait envoyé des « émissaires » pour les persuader de trancher des affaires politiques médiatisées conformément à ses préférences (David KOSAŘ, Jiří BAROŠ et Pavel DUFEK, "The Twin Challenges to Separation of Powers in Central Europe » European constitutional law review, n 15, vol. 3, p. 427-461, spéc. p. 456).

18. Tomasz Tadeusz KonCEWICZ, "Understanding the Politics of Resentment: of the Principles, Institutions, Counter-Strategies, Normative Change, and the Habits of Heart » in Indiana journal of global legal studies, 2019, vol. 26, $\mathrm{n}^{\circ} 2$, p. 501-630.

19. Thomas hochmann, « Cinquante nuances de démocrature ", Pouvoirs, 2019, n 169, p. 28.

20. Elle constituerait « une ligne de front potentielle contre les régimes autoritaires que ceux-ci ne peuvent accepter ». Philippe Xavier, "La légitimation constitutionnelle des démocratures", Pouvoirs, 2019, $\mathrm{n}^{\circ}$ 169, p. 39.

21. Adam CLINTON S. \& Mila VERSTEEG, « Court's limited ability to protect constitutionnal rights » in The University of Chicago law review, 2018, vol. 85, p. 293-336; Stephen GARDBAUM, «Are Strong Constitutional Courts Always a Good Thing for New Democracies?", Columbia journal of transnational law, 2015, $\mathrm{n}^{\circ}$ 53, p. 285-320.

22. Michaël HEIN, "Constitutional conflicts between politics and law in transition societies: A systems-theoretical approach » in Studies of transition states and societies, 2011, vol. 3, n 1, p. 3-23, cit. p. 3. Jasper DE RAADT, «Contested constitutions: legitimacy of constitution-making and constitutional conflict in central Europe ", East european politics and societies and cultures, vol. 23, $\mathrm{n}^{\circ} 3,2009$, p. 315-338.

23. Suivant une approche normative, le conflit constitutionnel est entendu comme un phénomène de concurrence interprétative entre deux pouvoirs constitués, qui porte sur la Constitution et qui se distingue par son mode de résolution juridictionnel. Autrement dit, le juge constitutionnel est présenté comme jouant un rôle de résolution et de prévention des conflits constitutionnels qui le place « en dehors » ou « en surplomb» du jeu de la séparation des pouvoirs (V. Élise CARPENTIER, «La résolution juridictionnelle des conflits entre organes constitutionnels », RIDC, vol. 59, 2007. p. 832. V. aussi : La résolution juridictionnelle des conflits entre organes constitutionnels, Paris, LGDJ, 2006 et « L'organe, l'acte et le conflit constitutionnel », AIJC, 2004, $\mathrm{n}^{\circ}$ 20, p. 57-91(cit. p. 85-86) : « Dès lors que les paramètres de référence applicables à un conflit institutionnel trouvent leur ancrage dans une disposition de la Constitution formelle relative à l'organisation du pouvoir, ou peuvent être regardés comme participant du droit constitutionnel matériel, le litige relève de la catégorie des conflits constitutionnels, et sa résolution ne peut ressortir qu'à la compétence de la juridiction constitutionnelle ". V. par ailleurs : Armel LE DIVELLEC, « Des conflits constitutionnels dans un État constitutionnel : le mécanisme des litiges entre organes devant la Cour constitutionnelle fédérale d'Allemagne » in Jacky HUMmel (dir.), Les conflits constitutionnels, Rennes, PUR, Col. « L'univers des normes », 2010, pp. 99-119). Suivant une approche politique du conflit constitutionnel, le conflit est également considéré comme un phénomène de concurrence interprétative entre deux pouvoirs constitués mais qui se distingue cette fois par son intensité et non plus son mode de règlement (Jacky HUMMEL, « Les conflits constitutionnels. Définition(s) d'un objet d'étude » in Jacky HUMMEL (dir.), Les conflits constitutionnels, op.cit.). Cette définition qui revalorise la dimension politique du droit constitutionnel contre une approche trop strictement contentieuse des phénomènes constitutionnels permet d'insister sur le fait que le pouvoir des juges constitutionnels est 
étroitement dépendant d'un ensemble de facteurs extra-juridique favorables qui font donc défaut dans un contexte illibéral.

24. Michel troper, Le Droit et la nécessité, Paris, PUF, 2011, p. 157.

25. Guillaume TUSSEAU, « Le Gouvernement [contraint] des juges... », loc.cit., p. 41-84, cit. p. 49.

26. Ibid., p. 48.

27. Ibid., p. 65 .

28. Ibid., p. 48.

29. Une telle étude pourrait par exemple s'attacher aux différents déterminants qui conditionnent le choix d'une ou de plusieurs stratégies par le juge constitutionnel, à la perception par la Cour de l'état du rapport de force qui l'oppose à l'interprète constitutionnel concurrent et de la capacité de réplique que ce dernier est susceptible de mettre en œuvre, aux stratégies internes aux cours constitutionnelles conduites en son sein par les différents juges, à la mise en œuvre, successives ou concomitante de plusieurs stratégies, à leurs conditions d'efficacité, autant de points qui excéderaient très largement le cadre d'analyse retenu ici.

30. Décision de la Cour constitutionnelle roumaine $n^{\circ} 727 / 2012$ du 9 juillet 2012 . V. le rapport de la commission de Venise CDL-REF_2012_031-e, Constitutional issues in Romania. Decisions, rulings and opinions of the constitutional court du 5 décembre 2012.

31. Arrêté du tribunal constitutionnel polonais $n^{\circ} 47 / 15$ du 14 janvier 2016 et Décision du tribunal constitutionnel polonais $\mathrm{n}^{\circ} \mathrm{K} 47 / 15$ du 9 mars 2016. V. Commission de Venise, CDL$\mathrm{AD}(2016) 001-\mathrm{f}$, Avis sur les amendements à la loi du 25 juin 2015 relative au Tribunal constitutionnel de Pologne, 11-12 mars 2016.

32. Décision de la Cour constitutionnelle hongroise $n^{\circ}$ 61/2011 (VII.13) du 12 juillet 2011.

33. V. Pierre-Alain collot, «Difficulté contre-majoritaire et usage impérieux du pouvoir constituant dérivé au regard de la quatrième révision de la Loi fondamentale de Hongrie ", RFDC, 2013/4 ( $\mathrm{n}^{\circ}$ 96), p. 789-812.

34. Décision de la Cour constitutionnelle hongroise $n^{\circ}$ 45/2012 (XII.29) du 29 décembre 2012.

35. Décision de la Cour constitutionnelle hongroise $n^{\circ} 13 / 2013$ (VI.17). V. à ce sujet: Eszter BODNÁR, « Reflections in the Practice of the Hungarian Constitutional Court on the Changes of Its Compétences » in Baller OESTEN, Breig BURKHARD (dir.), Justiz in Mittel- und Osteuropa. Berlin, Berliner Wissenschafts-Verlag, 2017, p. 154-156.

36. Décision de la Cour constitutionnelle roumaine $n^{\circ} 727$ du 9 juillet 2012, précit.

37. Voir supra.

38. Décision de la Cour constitutionnelle roumaine $n^{\circ} 738$ du 19 septembre 2012.

39. Décision I. ÚS 575/2016 du 6 décembre 2017. Voir CDL-REF(2017)013, Slovak Republic, Decisions by the Constitutional Court and further relevant texts (nota: La décision est mentionnée sous le numéro 757 et non 575) et CDL-AD(2017)001, avis sur des questions relatives à la nomination des juges à la Cour constitutionnelle, adopté par la Commission de Venise lors de sa 110e session plénière (Venise, 10-11 mars 2017). Sur cette décision, V. Simon DRUGDA, « Symposium on "The Slovak Constitutional Court Appointments Case"-Intermezzo to the Constitutional Conflict in Slovakia: À Case Critique ", I-connect, Blog of the international journal of constitutionnal law, 24 janvier 2018.

40. Le Président de la Cour constitutionnelle a ainsi déclaré devant les députés : "Je respecte l'indépendance et le libre exercice du mandat de chaque membre du Parlement. Par mon discours, je n'entends pas interférer de quelque manière que ce soit avec la liberté du mandat parlementaire. Je pense néanmoins que la connaissance de la situation actuelle de la Cour constitutionnelle peut utilement éclairer votre décision ».

41. V. Simon DRUGDA, Can the President of the Slovak Constitutional Court Defend It?, Int'l J. Const. L. Blog, July 2, 2019, at: http://www.iconnectblog.com/2019/06/can-the-president-of-the-slovakconstitutional-court-defend-it? 
42. Article S. 3 de la loi fondamentale hongroise.

43. V. supra.

44. Décision de la Cour constitutionnelle hongroise $n^{\circ} 12 / 2013$ du 24 mai 2013. Si la Cour a indiqué, dans un obiter dictum, qu'elle continuerait d'interpréter la Loi fondamentale comme un système unifié de valeurs, en prenant en compte les obligations de la Hongrie qui découle des traités internationaux, de l'adhésion à l'Union européenne et des règles du Droit international public, mais aussi les principes fondamentaux et les valeurs qui en découlent et qui sont intégrées dans la loi fondamentale, elle a toutefois pris soin de préciser qu'elle ne commentait pas, ce faisant, la relation spécifique des dispositions constitutionnelles en cause.

45. Dans une affaire pendante depuis plus de quatre ans, la Cour s'est finalement reconnue compétente pour exercer un contrôle substantiel des révisions constitutionnelles au regard d'un «socle » constitutionnel déduit de l'article premier de la Constitution. Sur ce fondement, elle a annulé un amendement à la Constitution qui visait à soumettre les candidats aux fonctions de juge de droit commun à un entretien de sécurité avant leur nomination. Toutefois, quelques mois plus tard, la Constitution a été amendée pour interdire explicitement à la Cour de contrôler les lois de révision constitutionnelle que ce soit sur le plan procédural ou substantiel. V. la décision de la Cour constitutionnelle slovaque n PL. ÚS 21/2014 du 30 janvier 2019. V. à propos de cette décision: Tomáš LALík, "The Slovak Constitutional Court on Unconstitutional Constitutional Amendment (PL. ÚS 21/2014)» in European Constitutional Law Review, 2020, n 16, vol. 2, p. 328-343; "Slovakia on its way to Illiberal Democracy: Nullifying the Power of the Constitutional Court to Review Constitutional Amendments » in I-connect Blog of the international journal of constitutional law, 18 décembre 2020, Marek Domin «A Part of the Constitution Is Unconstitutional, the Slovak Constitutional Court has Ruled», VerfBlog, 2 aout 2019, Drugda Simon, "Slovak Constitutional Court Strikes Down a Constitutional Amendment-But the Amendment Remains Valid", in I-connect Blog of the international journal of constitutional law, 25 avril 2019.

46. La juge Aspazia Cojocaru avait porté plainte en réaction aux menaces de mort dont elle était l'objet, V. Cosmina TAISONU, «Romania in the EU, political developments » in Amin VON BOGDANDY \& Pal SonNeVend (dir.), Constitutional Crisis in the European Constitutional Area, Hart/Beck, 2015, p. 178

47. Décision du tribunal constitutionnel polonais K 10/17 du 11 septembre 2017. V. infra.

48. V. not. les opinions dissidentes jointes aux décisions du Tribunal constitutionnel polonais $\mathrm{n}^{\circ} \mathrm{Kp} 1 / 17$ du 16 mars 2017 et K 5/17 du 20 juin 2017.

49. Résolution du 27 juillet 2017. V. Wojciech SADURSKI, « How Democracy Dies (in Poland) : A Case Study of Anti-Constitutional Populist Backsliding " in Sydney Law School Research Paper, 2018, vol. 18, $\mathrm{n}^{\circ}$ 1, p. 23-24 ; Benjamin BRICKER, « The (very) political dissent : Dissenting opinions and the Polish constitutionnal crisis », German Law Journal, 2020, n² 21, p. 1586-1605.

50. Rosalind DIXON \& Samuel ISSACHAROFF, « Living to fight another day: judicial deferral in defense of Democracy ", Wisconsin Law Review, 2016, n 4, p. 683-732.

51. Ibid.

52. Elena-Simina TĂNĂSESCU, « Roumanie » AIJC 2012, 2013, p. 924.

53. Samuel ISSACHAROFF, Fragile democracies: contested power in the era of constitutional courts, Cambridge, Cambridge University Press, 2015, p. 19.

54. Décision de la Cour constitutionnelle hongroise $n^{\circ} 3200 / 2018$ du 21 juin 2018. Voir Zoltán SZENTE and Fruzsina GÁRDOS-OROSZ, "Judicial deference or political loyalty? The Hungarian Constitutional Court's role in tackling crisis situations » in Zoltán SZENTE and Fruzsina GÁRDOSOROSZ (dir.), New challenges to constitutional adjudication in Europe (Routledge 2018), p.111-134. Eszter BODNÁR, Fruzsina GÁRDOS-OROSZ, Zoltán, POZSÁR-SZENTMIKLÓSY, « Hungary », 2018 Global Review of Constitutional Law, 2019, p. 138-140 ; Zoltán SZENTE, « The Political Orientation of Constitutional 
Judges in Hungary », Constitutional Studies, vol.1, p. 123-149. KÁLMÁN Pócza, Gábor DoBos, Attila GYULAI, "The Hungarian Constitutional Court: A constructive partner in constitutional dialogue ", in Kálmán PóczA (dir.), Constitutional Politics and the Judiciary. Decision-Making in Central and Eastern Europe, Routledge, 2018. Chapter 5.

55. Petra Léa LÁNCos, "Passivist Strategies Available to the Hungarian Constitutional Court ", ZaöRV- Heidelberg Journal of International Law, 2019, n 79, p. 971-993, spé. p. 984-988.

56. Décision du Tribunal constitutionnel polonais $n^{\circ} \mathrm{K} 1 / 18$ du 17 janvier 2019.

57. Décision du tribunal constitutionnel polonais K3/21 du 07 octobre 2021. V. infra.

58. Act $n^{\circ}$ LXXVI du 27 juin 2017.

59. Ordonnance de la cour constitutionnelle hongroise $n^{\circ}$ II/1460/2017.

60. Décision de la cour constitutionnelle hongroise $n^{\circ}$ 3048/ 2014 du 13 mars 2014. V. Petra Léa LÁNCOS, «Passivist Strategies Available to the Hungarian Constitutional Court », loc. cit., p. 990 ; Eszter BODNÁR, « All voters are equal but... », ICL Journal, vol. 10, n 4, 2016, p. 430-431.

61. Décision du Tribunal constitutionnel polonais $n^{\circ} \mathrm{K} 3 / 17$ du 24 octobre 2017

62. Décisions de la Cour constitutionnelle hongroise $n^{\circ} 6 / 2013$. (III. 1.) et $n^{\circ} 15 / 2019$ (IV. 17). V. Viktor Zoltán KAZAI, «Administrative Judicial Reform in Hungary: Who Gives a Fig about Parliamentary Process?», VerfBlog, 01 mai 2005 ; Viktor Zoltán KAZAI, «Le renforcement du contrôle de la procédure législative. Une stratégie proposée aux cours constitutionnelles opérant dans un système populiste ", AIJC 2018, 2019, nº 34, p. 765-781.

63. Décision de la Cour constitutionnelle hongroise $n^{\circ} 3 / 2019$ du 7 mars 2019.

64. Décision de la Cour constitutionnelle hongroise $n^{\circ} 1 / 17$ du 17 janvier 2017.

65. Décision de la Cour constitutionnelle hongroise $n^{\circ}$ 13/2017 du 19 juin 2017. V. Viktor Zoltán KAZAI, « Le renforcement du contrôle de la procédure législative », loc. cit.

66. V. infra

67. Décision du Tribunal constitutionnel polonais K5/17 du 20 juin 2017 et $n^{\circ} \mathrm{K} 12 / 18$ du 25 mars 2019.

68. Décision du Tribunal constitutionnel polonais K3/17 du 24 octobre 2017.

69. Lauriane ALLEZARD, «Juridictions constitutionnelles et populisme: vers un «autre» constitutionnalisme en Europe centrale?", AIJC 2019, n 34, 2018, p. 736.

70. Décision de la cour constitutionnelle hongroise $n^{\circ} 22 / 2016$, (XII. 5.) AB du 30 novembre 2016. V. infra.

71. "Hongrie : camouflet pour Orban, le Parlement rejette la révision constitutionnelle », Le Monde, 8 novembre 2016.

72. V. Wojciech SADURSKI, Poland's constitutional breakdown, Oxford university press, 2019, p. 82-83; «Polish Constitutional Tribunal Under PiS: From an Activist Court, to a Paralysed Tribunal, to a Governmental Enabler ", Hague Journal of the Rule of Law, $n^{\circ} 11,2019$, p. 63-84.

73. Article 24-2 d) de la loi fondamentale hongroise de 2011. Cette compétence était déjà présenté comme « une compensation » de la suppression de l'actio popularis.

74. Décision de la Cour constitutionnelle hongroise $n^{\circ} 3130 / 2018$ du 19 avril 2018.

75. Décision du Tribunal constitutionnel polonais K5/17 du 20 juin 2017 et $n^{\circ} \mathrm{K} 12 / 18$ du 25 mars 2019.

76. Décision du tribunal constitutionnel polonais K 10/17 du 11 septembre 2017

77. Décision du tribunal constitutionnel polonais $\mathrm{K} 9 / 17 \mathrm{du} 17$ juillet 2018

78. Jakub IWANIUK, « En Pologne, le pouvoir prend en main l'institution du médiateur des droits civiques ", Le Monde, 15 avril 2021

79. Décision du tribunal constitutionnel polonais Kpt $1 / 20$ du 21 avril 2020.

80. Décision de la cour constitutionnelle hongroise $n^{\circ} 22 / 2016$, (XII. 5.) AB. Pour justifier cette solution, la Cour s'appuie d'ailleurs, dans sa motivation, sur la citation de jurisprudences étrangères, en particulier la Cour constitutionnelle allemande, le Conseil constitutionnel et la 
CEDH. Sur cette décision V. not.: Jérémy BRZENCZEK «L'Union européenne et sa politique migratoire au défi de la "démocratie illibérale " hongroise : Chronique juridique du septième amendement à la Loi fondamentale et de la décision 22/2016 (XII.5.) AB de la Cour constitutionnelle hongroise ", Politeia, Automne 2017, n 32, p. 243-254 ; Zsolt KÖRTVÉLYESI, Balázs MAJTÉNYI, « Games of values : the threat of exclusive constitutional identity, the EU and Hungary » in German Law Journal, 2017, n 18, p. 1721-1744 ; Gábor HALMAI, « Abuse of Constitutional Identity. The Hungarian Constitutional Court on Interpretation of Article E) (2) of the Fundamental Law », in Review of Central and East European Law, 2018, n 43, vol. 1, p. 23-42 ; Ágoston монаY \& Tóth NORBERT, « Decision 22/2016. (XII. 5.) AB on the Interpretation of Article E)(2) of the Fundamental Law » in American Journal of International Law, 2017, vol. 111, n² 2, p. 468-475; Tímea DRINócZI, «Hungarian Constitutional Court: The Limits of EU Law in the Hungarian Legal System » ICL Journal, 2017, p. 139-151. Sur la notion d'identité constitutionnelle et son utilisation par les juges en Europe, V. en français : Laurence BURGORGUE-LARSEN (dir.), L'identité constitutionnelle saisie par les juges en Europe, Paris, Pédone, 2011: Danielle ROJAS, L'utilisation de la notion d'identité constitutionnelle, Institut francophone pour la justice et la démocratie, 2021.

81. Décision du tribunal constitutionnel polonais $\mathrm{K} 3 / 21 \mathrm{du} 07$ octobre 2021. V. not. En langue française sur cette décision : Francesco MARTUCCI, «La Pologne et le respect de l'État de droit: quelques réflexions suscitées par la décision $\mathrm{K} 3 / 21 \mathrm{du}$ Tribunal constitutionnel polonais ", Le club des juristes, 15 octobre 2021 ; Sébastien PLATON, « La décision du «tribunal » constitutionnel polonais sur la primauté du droit de l'union européenne. Une réponse à Jean-Eric SCHOETTL », RDLF, 2021, chrn. $\mathrm{n}^{\circ} 37$; Wojciech ZAGORSKI, « Quand la cour constitutionnelle polonaise réfute la jurisprudence de la cjue. Observations sous l'arrêt du 7 octobre 2021 », JP Blog, 21 octobre 2021 ; Florian REVERCHON, «Le nouvel arrêt du tribunal constitutionnel polonais sur l'application du droit européen : quelles conséquences juridiques?»,JP Blog, 19 octobre 2021 ;

\section{RÉSUMÉS}

Cet article se penche sur une forme paroxystique de concurrence interprétative en matière constitutionnelle: le «conflit» entendu comme la confrontation de deux prétentions concurrentes à l'interprétation constitutionnelle authentique. Cette définition permet de considérer le juge constitutionnel comme un protagoniste de tels conflits et d'envisager les stratégies que l'institution est susceptible de déployer pour y faire face en étudiant la politique jurisprudentielle de différentes cours constitutionnelles d'Europe centrale et orientale.

This paper deals with a paroxysmal form of interpretative competition in constitutional matters : the "conflict" understood as the confrontation of two competing claims to authentic constitutional interpretation. This definition makes it possible to consider the constitutional judge as a protagonist of such conflicts and to consider the strategies that the institution is likely to deploy in order to cope with them by studying the jurisprudential policy of various constitutional courts in Central and Eastern Europe. 
INDEX

Mots-clés : interprétation constitutionnelle, cour constitutionnelle, stratégie juridictionnelle, politique jurisprudentielle, Europe centrale et orientale, transition constitutionnelle, illibéralisme, régression démocratique

Keywords : constitutional interpretation, constitutional court, court strategy, judicial politics, Central and Eastern Europe, constitutional transition, illiberalism, democratic backsliding

\section{AUTEUR}

\section{ANTONIN GELBLAT}

Antonin Gelblat est maître de conférences en droit public à l'université de Rouen 